\title{
Comparing the emission spectra of $U$ and Th hollow cathode lamps and a new $U$ line list ${ }^{\star}$
}

\author{
L. F. Sarmiento ${ }^{1}$, A. Reiners ${ }^{1}$, P. Huke ${ }^{1}$, F. F. Bauer ${ }^{1}$, E. W. Guenter ${ }^{2,3}$, U. Seemann ${ }^{1}$, and U. Wolter ${ }^{4}$ \\ ${ }^{1}$ Institut für Astrophysik, Georg-August-Universität Göttingen, Friedrich-Hund-Platz 1, 37077 Göttingen, Germany \\ e-mail: sarmiento.luisfernando@gmail.com \\ 2 Thüringer Landessternwarte Tautenburg, Sternwarte 5, 07778 Tautenburg, Germany \\ 3 Instituto de Astrofísica de Canarias (IAC), 38205 La Laguna, Tenerife, Spain \\ ${ }^{4}$ Hamburger Sternwarte, Universität Hamburg, Gojenbergsweg 112, 21029 Hamburg, Germany
}

Received 21 February 2018 / Accepted 5 June 2018

\begin{abstract}
Context. Thorium hollow cathode lamps (HCLs) are used as frequency calibrators for many high resolution astronomical spectrographs, some of which aim for Doppler precision at the $1 \mathrm{~m} \mathrm{~s}^{-1}$ level.

Aims. We aim to determine the most suitable combination of elements ( $\mathrm{Th}$ or $\mathrm{U}, \mathrm{Ar}$ or $\mathrm{Ne}$ ) for wavelength calibration of astronomical spectrographs, to characterize differences between similar HCLs, and to provide a new U line list.

Methods. We record high resolution spectra of different HCLs using a Fourier transform spectrograph: (i) U-Ne, U-Ar, Th-Ne, and Th-Ar lamps in the spectral range from 500 to $1000 \mathrm{~nm}$ and $\mathrm{U}-\mathrm{Ne}$ and $\mathrm{U}-\mathrm{Ar}$ from 1000 to $1700 \mathrm{~nm}$; (ii) we systematically compare the number of emission lines and the line intensity ratio for a set of $12 \mathrm{U}-\mathrm{Ne}$ HCLs; and (iii) we record a master spectrum of U-Ne to create a new U line list.

Results. Uranium lamps show more lines suitable for calibration than Th lamps from 500 to $1000 \mathrm{~nm}$. The filling gas of the lamps significantly affects their performance because Ar and Ne lines contaminate different spectral regions. We find differences (up to $88 \%$ ) in the line intensity of $U$ lines in different lamps from the same batch. We find 8239 isolated lines between 500 and $1700 \mathrm{~nm}$ that we attribute to U, 3379 of which were not contained in earlier line lists.

Conclusions. We suggest using a combination of U-Ne and U-Ar lamps to wavelength-calibrate astronomical spectrographs up to $1 \mu \mathrm{m}$. From 1 to $1.7 \mu \mathrm{m}$, U-Ne shows better properties. The differences in line strength between different HCLs underline the importance of characterizing HCLs in the laboratory. The new 3379 U lines can significantly improve the radial velocity precision of astronomical spectrographs.
\end{abstract}

Key words. catalogs - line: identification - techniques: spectroscopic - methods: data analysis

\section{Introduction}

Thorium hollow cathode lamps (HCLs) are the de facto standard for wavelength calibration of astronomical high resolution spectrographs. The high number of spectral features emitted by HCLs from the cathode make these lamps good calibration sources for astronomical spectrographs. Current instrumentation used in searching for exoplanets (e.g. HARPS, Mayor et al. 2003 or CARMENES, Quirrenbach et al. 2014) use HCLs for wavelength calibration and achieve meter per second radial velocity precision. Commercially available HCLs fulfill most of the criteria of an ideal calibrator (Stanley \& Dieke 1955; Kerber et al. 2007; Lovis \& Pepe 2007): they are simple and safe to handle, are relatively inexpensive and readily available auxiliary equipment, and have a relatively long life time and narrow lines while covering a wide spectral range.

Since the first HCLs were introduced by Paschen (1916) as a light source for spectroscopic investigations, continuous improvements have been made. The first HCLs contained a vacuum circulating system, described by Tolansky (1947), to prevent impurities from outgassing, which shortened the life time

\footnotetext{
$\star$ The line list is only available in electronic form at the CDS via anonymous ftp to cdsarc.u-strasbg.fr (130.79.128.5) or via http://cdsweb.u-strasbg.fr/cgi-bin/qcat?]/A+A/618/A118
}

of the lamps. Further improvements of HCLs have been driven by the requirements of atomic absorption (AA) spectroscopy developed by Walsh in the 1950s. Dieke \& Crosswhite (1952) introduced an activated $U$ getter (consisting of a vaporized reactive metal layer deposited in the inner wall of the glass tube) for Fe cathode-based HCLs. This design prevents outgassing and yields a relatively long life time for sealed HCLs. Russell et al. (1957) presented zirconium or tantalum getters, which allowed HCLs of a wide variety of cathode materials to be sealed. Jones \& Walsh (1960) presented further improvements and detailed studies about the construction and characteristics of the sealed tubes used as a spectroscopic light source. With the introduction of the getter and the improvement of the glass tube to seal-off the HCLs, these lamps became commercially available. Since then, HCLs have been widely used in applications where narrow spectral lines are required, for example in the wavelength calibration of high resolution astronomical spectrographs.

Current commercial HCLs use this sealed design and contain a metal cathode, a metal anode, and a buffer gas at a defined pressure. The metal cathode can be either made of a single species or of a combination of different elements. The buffer gas must be a nobel gas to avoid a chemical reaction with the cathode material, which would contaminate the spectrum with molecular emission lines. The front window of the glass tube 
must be transparent in the wavelength region for which it is used. The physical principles of operating HCLs have been studied in great detail by different authors (Crosswhite et al. 1955; Lieberman \& Lichtenberg 2015; Huke et al. 2016). As explained in Kerber et al. (1960), HCLs are operated by applying a voltage between the cathode and the anode. Therefore, an electric potential is established between them. The negative potential of the cathode accelerates the electrons towards the anode. During this process, their energy increases until they have sufficient energy to ionize the neutral buffer gas atoms through inelastic collisions that create a hot plasma. The cations from the plasma accelerate towards the cathode, colliding with its surface at energies high enough to overcome the electronic work function of the metal cathode. Material from the cathode is sputtered at high velocities, usually in the form of unexcited atoms. The sputtering rate depends on the cathode material, the mass of the buffer gas, and the voltage applied between the cathode and the anode. The sputtered material collides with fast electrons ionizing the metal material, and with atoms from the buffer gas, resulting in unstable excited states. The metal and gas atoms relax back to the ground state and therefore emit photons during the process. Therefore HCLs yield spectral features from two different sources: the material of the cathode and the buffer gas. The intensitiy of the lines is dependent on the operational current. Higher operational current yields a higher voltage difference between the cathode and the anode. The cations from the buffer gas hit the cathode with higher energies, which in turn increases the rate of sputtered material from the cathode (Kerber et al. 1960). Therefore, not only are more metallic lines emitted, but metal lines also grow faster in intensity than buffer-gas-emitted lines.

To use HCLs as calibrators for astronomical spectrographs, the wavelength of the spectral lines must be known with high accuracy and precision. Therefore, HCLs are measured in laboratories using Fourier transform spectrographs (FTS), which offer the required precision and accuracy to carry out these interferometric measurements covering a wide spectral range. Great efforts have been made during the last decades to create line lists for different cathode elements. The Th line list presented by Redman et al. (2014) (from now on R2014) combines precise FTS measurements of Th-Ar HCLs with seven previous works (Giacchetti et al. 1974; Zalubas \& Corliss 1974; Zalubas 1976; Palmer \& Engleman 1983; Engleman et al. 2003; Lovis \& Pepe 2007; Kerber et al. 1960) to calculate accurate Ritz wavelengths.

Pure Th cathode lamps have not been manufactured for several years (Fischer et al. 2016). Current Th HCLs contain a cathode made out of Th-oxide, which introduces impurities into the cathode. High resolution measurements of the Th-oxide lamp's spectrum show undesirable spectral features, also known as a "grass" of unidentified emission lines. These features compromise the wavelength calibration of high accuracy radial velocity spectrogaphs. Uranium HCLs have been proposed to be used for high precision wavelength calibration in the near infrared (NIR) spectral range in Redman et al. (2011; from now on R2011), where a line list was published as a line list from 0.85 to $4 \mu \mathrm{m}$. R2011 complements previous works: Palmer et al. (1980) created a high energy line list from 3846 to $9091 \AA$, and Conway et al. (1984) published a line list from 1.8 to $5.5 \mu \mathrm{m}$. However, R2011 has a reduced number of lines in the region around $1080 \mathrm{~nm}\left(9250 \mathrm{~cm}^{-1}\right)$ because of the low sensitivity of the 1-m FTS of the Kitt Peak National Solar Observatory in that band. This spectral region overlaps the $Y$ band, which is of special interest for astronomical observations of M dwarfs (Reiners et al. 2010).

Because pure Th cathode lamps are currently not available, and there are no line lists available for alternative cathode materials, wavelength calibration of optical spectrographs becomes increasingly difficult. We explore if $\mathrm{U}$ can also be an alternative for the established Th cathodes in the wavelength range from 500 to $1000 \mathrm{~nm}$ (like in the NIR as proposed by Redman et al. 2011). Uranium cathode HCLs are made of natural $\mathrm{U}$ with isotope abundances ${ }^{238} \mathrm{U} \sim 99.3$ and ${ }^{235} \mathrm{U} \sim 0.7 \%$ and a few additional isotopes with abundances smaller than $0.01 \%$ (Rosman \& Taylor 1998). These isotopes may contribute to lines we identify as $U$ lines as well as to the unidentified lines found in our spectra. However, the second most abundant isotope, ${ }^{235} \mathrm{U}$ is hyperfine-structured. Therefore, the expected line intensities are low and the contribution to the observed U spectra can be neglected (Redman et al. 2011). We devote this work to the performance comparison between the currently available Th and $U$ lamps and present a uniform $\mathrm{U}$ line list spanning the wavelength range from 500 to $1700 \mathrm{~nm}$.

In Sect. 2, we explain our experimental setup and our FTS instrument used to take high resolution spectra of the hollow cathode lamps. In Sect. 3, we identify spectral features in the spectra of four HCLs: U-Ne, U-Ar, Th-Ne, and Th-Ar. Then, we match the features with previously published line lists and determine the emitting element of the lines found in our spectra. In Sect. 4, we discuss the performance of each of the four lamps. In Sect. 5, we briefly discuss the finding of molecular bands in the spectra of the Th-Ar lamp. Because HCLs are replaced on a regular basis in observatories, a similar performance for lamps of the same type is desired. In Sect. 6, we test 12 individual U-Ne lamps to identify how large the differences between lamps of the same production batch can be. Finally, our U line list is presented in Sect. 7. Section 8 summarizes our results.

\section{Experimental setup and measurements}

The lamps used in this work are currently used in the Calar Alto high-Resolution search for $\mathrm{M}$ dwarfs with Exoearths with Near-infrared and optical Échelle Spectrographs (CARMENES) (Quirrenbach et al. 2014) calibration unit. For this purpose 12 identical U-Ne lamps were ordered from Photron PTY Ltd. ${ }^{1}$. The HCLs belong to the same batch and have consecutive serial numbers (to ensure that they were built in a similar process). To record high quality spectra and operate the lamps safely, HCLs must be placed in a housing. It was designed and built by the Thüringer Landessternwarte Tautenburg workshop.

To feed the light from the glowing cathode into the spectrograph (via a fibre) we used the fore-optics shown in Fig. 1. It consists of: L1, a doublet to collimate the light from the cathode located at a distance to the lamp front window (LL1) of $35.5 \mathrm{~mm}$; a second doublet L2, located at a distance to the doublet L1 of $60.3 \mathrm{~mm}$ (LL2), which feeds the light into the fibre using a SM1SMA-SMA fibre adaptor. The distance LL3, between the doublet L2 and the fibre adaptor is $75 \mathrm{~mm}$ (for further details see the caption of Fig. 1). We connected a power-meter to the fibre port to have a simple method for aligning and focusing the light source.

We equipped our FTS source chamber with a 8:92 (R:T) Thorlabs ${ }^{1}$ pellicle beam splitter and a photodiode. The beam splitter reflected $8 \%$ of the incident light into a photodector, which measured the flux with a frequency of $1 \mathrm{kHz}$. Simultaneously, we measured the operational voltage of the lamp using a Universal Serial Bus (USB) voltmeter with a frequency of $2 \mathrm{~Hz}$.

\footnotetext{
1 The identification of commercial products in this document intends to specify the experimental procedure properly. It is not intended to imply recommendation or endorsement, nor is it intended to imply that the products identified are necessarily the best available for the purpose.
} 


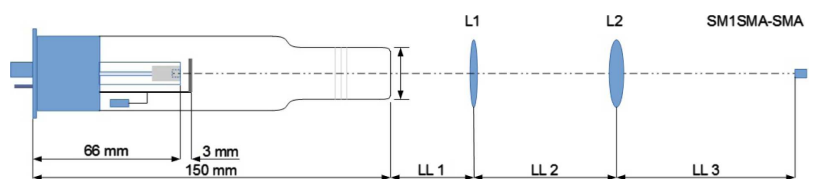

Fig. 1. Light from the HCL is coupled into a fibre using a set of optics: two doublets designated by L1 (AC254-125-B-ML from Thorlabs ${ }^{1}$ ), L2 (AC254-075-B-ML from Thorlabs ${ }^{1}$ ), and a SM1SMA-SMA fibre adaptor. The text provides further details.

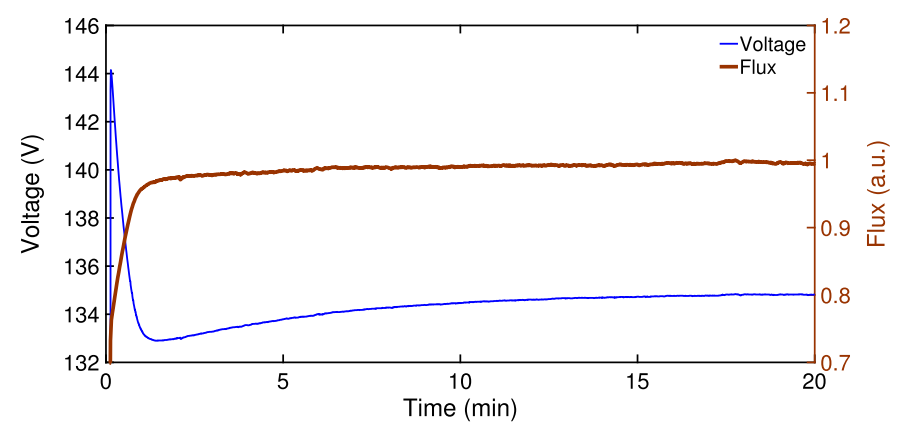

Fig. 2. Example of flux (brown) and voltage (blue) during the lamp warm-up time of one of our U-Ne lamps.

Figure 2 shows the measured voltage (blue) and flux (brown) during $20 \mathrm{~min}$ after switching on one of our lamps. The changes in both parameters, voltage and flux, indicate that the HCL needs a warmup time to produce a stable output. To analyse these changes, we first averaged the voltage and flux measurements every minute. In a second step, we calculated the slope of the relative voltage variation and the relative flux variation of the averaged values. The changes in flux were less than $0.5 \%$ after $7 \mathrm{~min}$. However, we observed changes in voltage within approximately $14 \mathrm{~min}$, before the voltage reached a stability of $0.05 \%$. Therefore we recommend a minimum warm-up time of the Photron HCLs of 15 min to get the most stable output (see Fig. A.1 in Appendix A).

We recorded the spectra of the lamps using our IFS125HR FTS. In Table A.1, we summarize the resolution, number of scans, and operational current used for the different experiments. To obtain high quality spectra that cover the full CARMENES spectral bandwidth from 500 to $1700 \mathrm{~nm}$ (from 20000 to $5882.4 \mathrm{~cm}^{-1}$ ), we used two different setups. For the following, we call the first setup the visible (VIS) one. We recorded spectra from 500 to $1000 \mathrm{~nm}$ (from 20000 to $10000 \mathrm{~cm}^{-1}$ ) using a quartz beam splitter and a silicon diode detector. The second setup, called the NIR setup, was used to record spectra from 1000 to $1700 \mathrm{~nm}$ (from 10000 to $5882.4 \mathrm{~cm}^{-1}$ ). The NIR setup consists of a calcium fluoride beam splitter and an InGaAs detector.

Our FTS uses a frequency-stabilized HeNe laser (at a wavelength of $632.816 \mathrm{~nm}$ in air, equivalent to $15802.382 \mathrm{~cm}^{-1}$ ) to obtain the internal wavenumber solution by measuring the optical path difference of the interferometer during the mirrors scanning. A fraction of the laser light is reflected during its path and also enters the detector. Hence, the VIS FTS spectra used for this work are strongly contaminated by the laser in the region between 610 and $655 \mathrm{~nm}$ (from 16393.4 to $15267.2 \mathrm{~cm}^{-1}$ ). Therefore, we are less sensitive to weak spectral lines in the contaminated range, and we can only detect the strongest emission lines in our spectra (Table A.2).

\section{Line search, line identification, and spectrum calibration}

\subsection{Identification of line candidates}

The sensitivity of the FTS in combination with the HCL light source yields a low signal to noise ratio $(\mathrm{S} / \mathrm{N})$ spectrum in single scans. To increase the signal, we averaged over a high number of individual scans (see Table A.1 in Appendix A).

To quantify the noise level of the spectrum, we excluded the points with the highest intensities (10\%) and calculated the standard deviation and the mean of the remaining signal; we defined our noise level as $\mu+(3 \times \sigma)$. Next, we defined a wavenumber bandwidth of $0.09 \mathrm{~cm}^{-1}$ around each point with intensity higher than the defined noise levels, and selected the data points that have a continuous first derivative centred on the zero crossing and flagged them as "detected line". We excluded the points with intensities lower than the noise level to determine better line parameters. Finally, we fitted a Gaussian curve to each emission line and calculated the centre of the line, the maximum intensity, and the full width at half maximum (FWHM). To estimate the uncertainties of these parameters, we first obtained the statistical uncertainties of the fitted parameters from the covariance matrix. We then applied error propagation to calculate the uncertainties of the centre of the lines, their maximum intensity, and the FWHM.

\subsection{Lines found in the literature}

To determine the emitting element for the detected lines, we first looked for a match of these lines with a line in the literature. Next, we confirmed the line identification by analysing the FWHM of the line.

We calculated the wavenumber difference between the line position of our detected lines and lines from the R2011 line list to identify U lines, and the R2014 line list to identify Th lines. We used the NIST line list (Kramida et al. 2015) to identify $\mathrm{Ne}$ and Ar lines. Detected lines that match a line position listed in the line lists within three times its estimated position uncertainty were flagged as "confirmed" lines.

Lines emitted by the metallic material of the cathode (either $\mathrm{U}$ or $\mathrm{Th}$ ) and the lines emitted by the HCL's filling gas (either Ne or Ar) have very different widths (Doppler broadening) because of their different atomic weight (Th:232 u, U:238 u, Ne:20 u, Ar:40 u). Hence, the lines emitted by $U$ or Th are narrower than the lines emitted by $\mathrm{Ne}$ or Ar. The possibility that the lines emitted by the buffer gas and the cathode have different FWHM is used to confirm the line identification done by matching the line position. In Fig. 3, we show that the FWHMs of metal and gas lines differ significantly between $\mathrm{U}$ and $\mathrm{Ne}$ lines found in our NIR spectrum.

We fitted the FWHM of the confirmed lines as a function of wavenumber and used this information to determine the emitting element for the remaining detected lines in Sect. 3.3. We computed the residuals to the fit and calculated the standard deviation of the residuals. Lines with residuals higher than three times the standard deviation were flagged as blends, while lines with residuals smaller than three times the standard deviation were flagged as "isolated" lines.

Before classifying the lines that were not found in the literature, we searched for the traces of additional contaminants that might be present in our recorded spectra. We matched the line position of the unidentified lines with the lines in the line lists of $\mathrm{Ne}$ (in lamps where Ar is used as the filling gas), $\mathrm{Ar}$ 


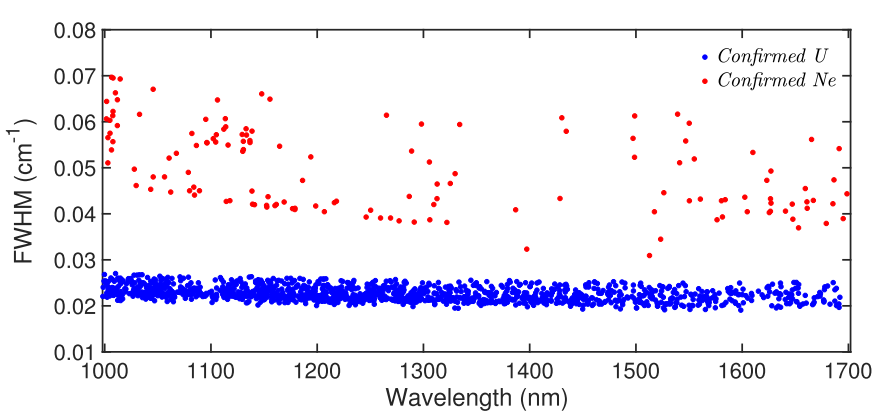

Fig. 3. FWHM of confirmed U (blue dots) and confirmed Ne (red dots) lines in the spectrum of one of our U-Ne lamps.

(in lamps where $\mathrm{Ne}$ is used as the filling gas), $\mathrm{N}$ (because it is the most abundant gas in the atmosphere), and $\mathrm{Zr}$ (because it is used in the lamps tested here to trap molecules sputtered from the cathode) that were downloaded from the National Institute of Standards and Technology (NIST) database (Kramida et al. 2015). We did not find any significant gas contamination in our measurements. As a result of the first line identification analysis, $87 \%$ of the emission lines remained unidentified in the VIS spectrum, and 50\% in the NIR spectrum (see Tables A.3 and A.4 in Appendix A). In Sect. 3.3, we attempt to classify the large number of remaining unidentified lines to build a list of $U$ line candidates.

\subsection{Identification of new $U$ line candidates}

Since we found a large number of unidentified lines in our spectra, we are interested in identifying the lines emitted by the metallic cathode. In a first step, we selected those lines from the "unidentified detected lines" that have measured FWHM smaller than the confirmed $\mathrm{Ne}$ lines. Next, we checked that those lines are in line with the fitted FWHM $(v)$ relation determined from the confirmed $U$ lines. The lines that matched both criteria were flagged as "U line candidates". To confirm the nature of the U line candidates, we will examine the lines further in Sect. 7. In that section, we will analyse the line intensity, operating the lamps at different currents to confirm the emitter element (as in Kerber et al. 2007).

\subsection{Spectrum calibration}

\subsubsection{Wavenumber calibration}

Our FTS wavenumber solution is based on an internal frequencystabilized He-Ne laser. The laser is used to measure the optical path difference of the interferometer during measurements. Any misalignment between the light path of the science light and the internal laser combined with the limited size of the FTS entrance aperture introduces a linear compression or stretch of the instrument wavenumber scale (Griffiths \& de Haseth 2007). We corrected for this effect using eight $\mathrm{U}$ and seven Th standard lines between 694 and $755 \mathrm{~nm}$ (Degraffenreid et al. 2002). The wavenumber of the standard lines was measured with an accuracy of the order of $10^{-8}\left(0.0002 \mathrm{~cm}^{-1}\right)$, which is one order of magnitude higher than the accuracy of our measured line positions $\left(10^{-7}\right)$. The wavenumber correction factor was calculated for each standard line $i$ as

$k_{i}=\frac{\tilde{v}_{\mathrm{std}, \mathrm{i}}}{\tilde{v}_{\mathrm{obs}, i}}-1$,

A118, page 4 of 14 where $\tilde{v}_{\mathrm{std}, i}$ is the standard wavenumber of line $i$ and $\tilde{v}_{\mathrm{obs}, i}$ is our measured wavenumber. We calculated the weighted average of the correction factor of all the lines to obtain the spectrum wavenumber correction factor $k$. To convert the FTS wavenumber scale into an absolute scale, we computed

$\tilde{v}_{\mathrm{c}}=(1+k) \times \tilde{v}_{\mathrm{FTS}}$,

where $\tilde{v}_{\text {FTS }}$ is the internal FTS wavenumber scale and $\tilde{v}_{\mathrm{c}}$ is the corrected wavenumber scale.

We obtained $k_{\mathrm{VIS}}=(1.4 \pm 0.1) \times 10^{-7}$ for the spectrum measured in the VIS band. No Th or U standard lines are available similar to the ones of Degraffenreid et al. (2002) for our NIR setup. Therefore, we used 21 confirmed and isolated, high intensity $U$ lines that we found in the wavelength region where our VIS setup and NIR setup overlap to cross calibrate our two setups. The errors estimated for these lines in the VIS spectrum are of the order of $0.001 \mathrm{~cm}^{-1}$. Hence, each single line can be measured with a precision comparable to the expected wavenumber correction (see Fig. A.3). Using the weighted average of the $21 k_{i}$, we obtained $k_{\mathrm{NIR}}=(-2.85 \pm 0.9) \times 10^{-7}$. We noted that the higher uncertainty of the $k_{\mathrm{NIR}}$ was a result of using the 21 absolute calibrated lines from the VIS setup, which are less accurate than the standards lines of Degraffenreid et al. (2002) used to calibrate the VIS spectrum.

\subsubsection{Flux calibration}

We calibrated the line intensity for the spectral response of the FTS and the fore-optics. For this, we recorded a low resolution spectrum of a calibrated tungsten lamp (with a black body temperature of $3000 \mathrm{~K}$ ). We applied a moving average to obtain the continuum of the spectrum and divided the continuum curve by the theoretical black body curve of the lamp. We identified the calculated curve with the spectral response of the setup. The line intensity of each line was corrected by dividing the calculated line intensity by the spectral response curve.

\section{Cathode and buffer gas elements selection}

In this section, we characterize U-Ne and U-Ar lamps and compare them with Th-Ne and Th-Ar lamps (from 500 to $1000 \mathrm{~nm}$ ). Since the number of Th lines is known to drop significantly beyond $1000 \mathrm{~nm}$ (Redman et al. 2014), we restricted our analysis for the NIR setup (from 1000 to $1700 \mathrm{~nm}$ ) only to the U-Ar and U-Ne lamps.

To characterize all lamps, we quantified the number of isolated metal and gas lines and analysed their spectral distribution. Another important characteristic of HCLs when used in astronomical spectrographs is that high intensity lines can produce saturation effects in the cgarge-coupled device (CCD). These lines are mostly emitted by the buffer gas of the HCLs. Therefore, the number of these high intensity lines and their spectral distribution should be identified.

\subsection{Analysis in the VIS}

We systematically analysed the metal and gas lines of U-Ar, $\mathrm{U}-\mathrm{Ne}$, Th-Ar, and Th-Ne HCLs using the VIS setup. We averaged 200 scans per lamp taken under the same conditions (same imaging fore-optics and $12 \mathrm{~mA}$ operational current) at a resolution of $0.03 \mathrm{~cm}^{-1}$. For our analysis, we identified the metal (U and $\mathrm{Th}$ ) and buffer gas ( $\mathrm{Ne}$ and $\mathrm{Ar}$ ) lines emitted by all four HCLs as explained in Sect. 3.2. The number of confirmed metal and gas lines in all four lamps is summarized in Table 1. In the

A118, page 4 of 14 
Table 1. Confirmed metal and gas lines using the VIS setup for the experiment cathode and buffer gas elements selection.

\begin{tabular}{lccc}
\hline \hline $\begin{array}{l}\text { HCL } \\
\text { elements }\end{array}$ & $\begin{array}{c}\text { Metal } \\
\text { lines }\end{array}$ & $\begin{array}{c}\text { Gas } \\
\text { lines }\end{array}$ & $\begin{array}{c}\text { High intensity } \\
\text { gas lines }\end{array}$ \\
\hline U,Ne & 1899 & 235 & 43 \\
U,Ar & 1998 & 195 & 26 \\
Th,Ne & 938 & 231 & 66 \\
Th,Ar & 1436 & 233 & 26 \\
\hline
\end{tabular}

Notes. High intensity gas lines are defined as the gas lines with intensities higher than the most intense metal lines.

last column of Table 1, we show the number of "high intensity gas lines", which we define as the gas lines with intensities higher than the most intense metal line.

In the second column of Table 1, we see a larger number of confirmed lines in the U HCLs than in the Th HCLs. The number of confirmed isolated U lines in the U-Ar spectrum (1998) is slightly larger than in the U-Ne spectrum (1899), but both numbers are still comparable. However, they are very different for the Th lamps: while we identify 1436 isolated Th lines in the Th-Ar lamp spectrum, we only find 938 isolated Th lines in the spectrum of the Th-Ne lamp. Comparing the Th-Ar and U-Ar lamps we observe the same number of confirmed high intensity Ar lines in both spectra. However, when we compare the number of confirmed high intensity $\mathrm{Ne}$ lines in the Th-Ne with the U-Ne lamps, we find significantly fewer strong $\mathrm{Ne}$ lines in the $\mathrm{U}-\mathrm{Ne}$ lamp.

Besides the total number of metal lines, their spectral distribution is also important, as explained in Sect. 1. Therefore, we plot the histogram of the number of confirmed metal lines as a function of wavelength in Fig. 4 for all four lamps. We also indicate the confirmed high intensity gas lines as vertical lines.

We first discuss the upper panel of Fig. 4 by comparing the Th-Ar and Th-Ne lamps. We see that there are fewer confirmed Th lines in the Th-Ne lamp throughout the entire spectral range of the VIS setup. The difference in the number of confirmed Th lines between $\mathrm{Th}-\mathrm{Ar}$ and $\mathrm{Th}-\mathrm{Ne}$ is relatively homogeneously distributed. The number of confirmed gas lines, however, is comparable in both lamps (233 in Th-Ar and 231 in Th-Ne) although the Ne lines show higher intensities than Ar lines. Twenty-six of the Ar lines in the Th-Ar lamp are high intensity gas lines. For the Th-Ne lamp we count 66 high intensity gas lines, which is more than a factor of two higher than in the Th-Ar lamp. It is interesting to note that high intensity $\mathrm{Ne}$ lines are mainly emitted at wavelengths shorter than $750 \mathrm{~nm}$, while the high intensity $\mathrm{Ar}$ lines are emitted at wavelengths longer than $695 \mathrm{~nm}$. From the total number of Th lines and their spectral distribution, we conclude that $\mathrm{Th}-\mathrm{Ar}$ is a better choice for wavelength calibration than $\mathrm{Th}-\mathrm{Ne}$ for any spectrograph operating in the range from 500 to $1000 \mathrm{~nm}$.

After analysing the Th cathode lamps, we take a close look at the lower panel of Fig. 4 and compare U-Ar with U-Ne. The number of confirmed $\mathrm{Ne}$ lines in the U-Ne spectrum (235) is slightly higher than the confirmed Ar lines (195) in the U-Ar lamp. Moreover, we find 43 high intensity Ne lines, which is a factor of 1.6 larger than the number of high intensity Ar lines (26). Most of the high intensity Ne lines are emitted at short wavelengths (shorter than $750 \mathrm{~nm}$ ) while Ar lines are mostly emitted at wavelengths longer than $695 \mathrm{~nm}$, as we previously saw for the Th cathode lamps. We identify a slightly larger number of confirmed metal lines and a lower number of high intensity lines and conclude that U-Ar performs better than U-Ne HCLs in the VIS band.

After comparing lamps with the same cathode material, our next goal is a comparison of different cathode materials, Th and $\mathrm{U}$, regarding their usefulness for calibration purposes in the VIS spectral range (from 500 to $1000 \mathrm{~nm}$ ). The position of brighter lines can be determined with higher accuracy, and a high number of calibration lines in the spectra is desirable for computing the wavelength solution of astronomical spectrographs. To account for both, we define the quality factor of a lamp as the sum of all line intensities in a given spectral range.

To compare lamps with this method, we normalized the spectra by the intensity of the gas lines because the intensity of these lines typically limits the exposure time of a detector. We calculate the normalizing factor of the Ar HCLs spectra using the 26 high intensity Ar lines identified in the U-Ar and Th-Ar spectra, and the normalizing factor of the Ne HCL spectra using the 43 high intensity $\mathrm{Ne}$ lines in the spectra of the $\mathrm{U}-\mathrm{Ne}$ and $\mathrm{Th}-\mathrm{Ne}$ HCLs. After normalizing the spectra, the total line intensity is computed over spectral ranges of $45 \mathrm{~nm}$ bins.

In Fig. 5 the uppel panel depicts the results of the HCLs with Ar buffer gas and the bottom panel shows the results of the HCLs with $\mathrm{Ne}$ buffer gas. The results from $\mathrm{U}-\mathrm{Ar}$ and $\mathrm{Th}-\mathrm{Ar}$ are comparable in the first two wavelength bins from 500 to $591 \mathrm{~nm}$ and in the last two wavelength bins from 909 to $1000 \mathrm{~nm}$. For the rest of the VIS spectra (with the exception of the central wavelength range from 636 to $681 \mathrm{~nm}$ ), the U-Ar lamp clearly outperforms the Th-Ar lamp. This is also reflected in the total line intensity when summed over the entire VIS spectral range. Overall, the $\mathrm{U}$ lines in the U-Ar spectrum exhibit $16 \%$ more calibration light than the Th lines in the Th-Ar spectrum. For the lamps with Ne buffer gas (bottom panel in Fig. 5), we see that the total line intensity per spectral range is higher in almost the entire VIS spectral range for the U-Ne lamp. The only exception is the wavelength bin centred at $660 \mathrm{~nm}$, where U-Ne and Th-Ne are comparable. The total amount of light available in the $U$ lines of the U-Ne lamp is $222 \%$ greater than the total amount of light available in the Th lines of the Th-Ne lamp.

From our results we conclude that, in the defined VIS spectral range (from 500 and $1000 \mathrm{~nm}$ ), U HCLs are more suitable for wavelength calibration of high resolution spectrographs than Th HCLs. Among the U lamps, we identify a slightly higher number of confirmed isolated cathode lines in the U-Ar than in $\mathrm{U}-\mathrm{Ne}$ spectrum. Although both lamps show a considerable number of filling gas lines, the high intensity gas lines are more numerous and also brighter in the U-Ne HCL. The high intensity lines can saturate the CCD of the spectrographs, which can contaminate extended regions of the spectrum and make them useless for wavelength calibration. Therefore, U-Ar lamps show better properties than U-Ne HCLs for wavelength calibration.

To improve the wavelength solution in the spectral regions of strong gas lines ( $\mathrm{Ar}$ or $\mathrm{Ne}$ ), one strategy is to use both the $\mathrm{U}-\mathrm{Ar}$ and $\mathrm{U}-\mathrm{Ne}$ lamp independently. Uranium lines blended with strong gas lines in one of the lamps will be uncontaminated in the other because $\mathrm{Ne}$ and Ar emit lines in different spectral regions. The $\mathrm{U}$ lines found in both spectra can then be combined and used for wavelength calibration. This strategy is currently used in the VIS channel of the CARMENES spectrograph.

\subsection{Analysis in the NIR}

After analysing the VIS setup, we performed the same analysis for U-Ne and U-Ar HCLs in the NIR wavelength range (from 


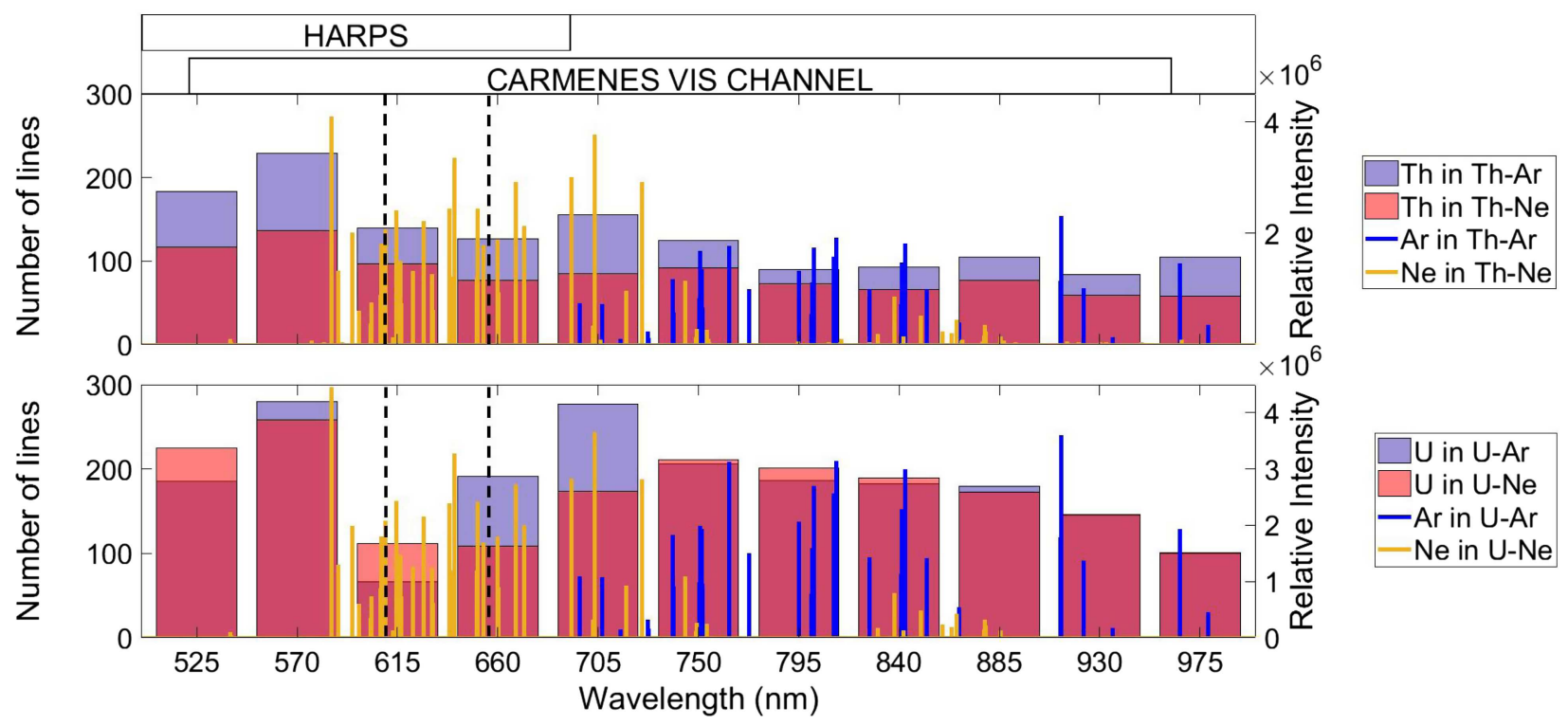

Fig. 4. Number of metal lines per $45 \mathrm{~nm}$ (light blue for Ar buffer gas HCLs and light red for Ne buffer gas lamps) using VIS setup. The area where both overlap is represented in magenta. The high intensity gas lines (i.e. gas lines with intensities higher than the most intense metal line) are overplotted. Argon lines are represented in blue and Ne lines in orange. In the top part, we indicate the spectral range covered by the CARMENES VIS channel and HARPS (Mayor et al. 2003). The spectral region between the dashed lines (from 610 to $655 \mathrm{~nm}$ ) indicates where the internal laser of our FTS contaminates the recorded spectra. Upper panel: Th cathode HCLs (Th-Ne and Th-Ar). Lower panel: U cathode HCLs (U-Ne and U-Ar). Top part of the plot shows the spectral range coverage of high resolution astronomical spectrographs. The spectral region within the dashed lines indicates where the line identification is affected by the internal FTS laser (see Sect. 2).

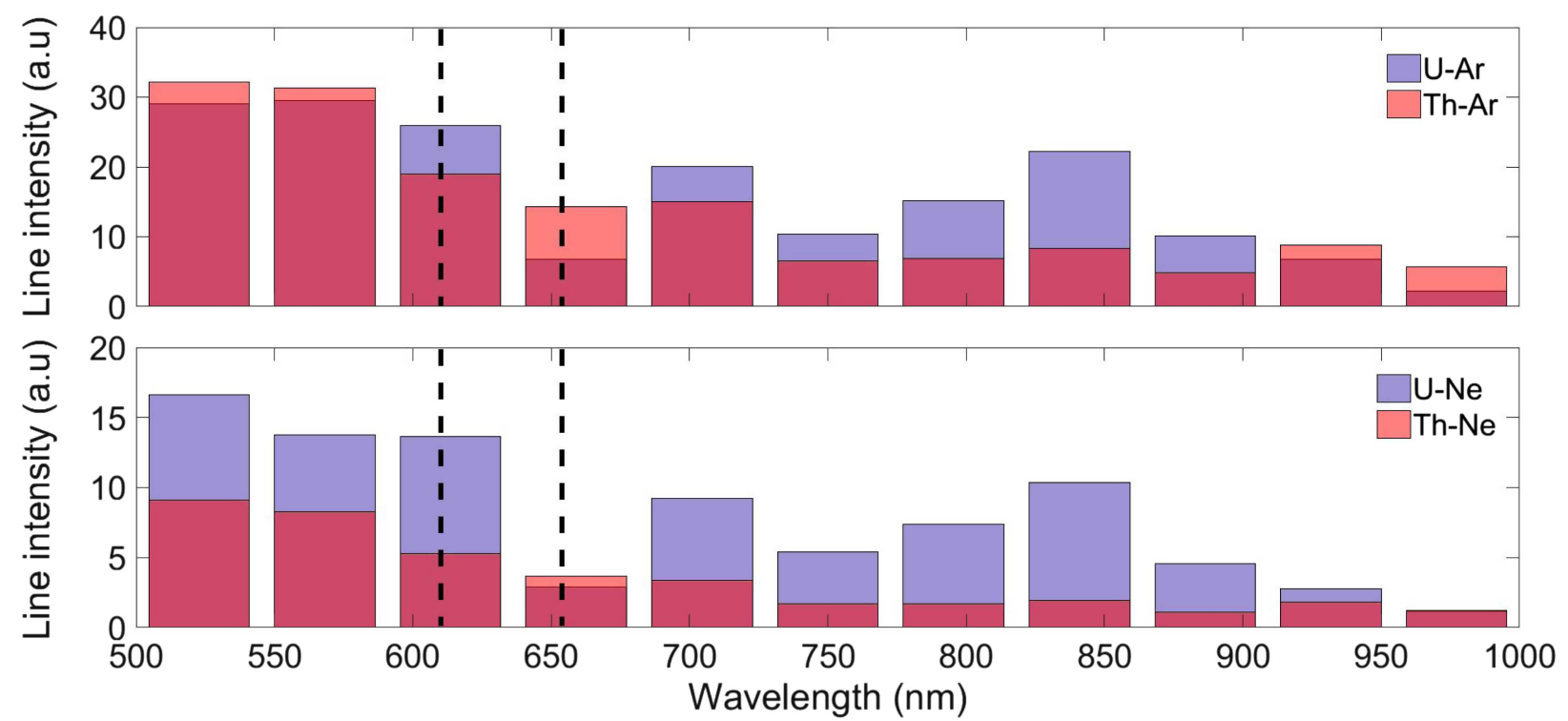

Fig. 5. Total line intensity of metal lines per $45 \mathrm{~nm}$ in VIS setup. Blue bars represent the total line intensity per spectral range of U lines and red represents Th lines. Magenta indicates the overlap areas. The spectral region between the dashed lines (from 610 to $655 \mathrm{~nm}$ ) indicates where the internal laser of our FTS contaminates the recorded spectra. Upper panel: HCLs with Ar buffer gas (U-Ar and Th-Ar). Bottom panel: HCLs with Ne buffer gas (U-Ne and Th-Ne).

1000 to $1700 \mathrm{~nm})$. We averaged 200 scans taken at a resolution of $0.02 \mathrm{~cm}^{-1}$ while the lamps were operated at $12 \mathrm{~mA}$. We started by identifying the $\mathrm{U}$ and buffer gas lines. The number of confirmed $\mathrm{U}$ and $\mathrm{Ne}$ lines, and the high intensity gas lines found in the two spectra, are summarized in Table 2.

From Table 2, we can see that the number of confirmed U lines in the U-Ne HCL (1538) is slightly higher than in the U-Ar lamp (1480). Although we identify a similar number of confirmed gas lines (188 Ne and 179 Ar lines), the number of high intensity Ar lines (31) is about a factor of two larger than the number of $\mathrm{Ne}$ lines (15).
Table 2. As in Table 1, but for U cathode lamps.

\begin{tabular}{lccc}
\hline \hline $\begin{array}{l}\text { HCL } \\
\text { elements }\end{array}$ & $\begin{array}{c}\mathrm{U} \\
\mathrm{U}\end{array}$ & $\begin{array}{c}\text { Gas } \\
\text { lines }\end{array}$ & $\begin{array}{c}\text { High intensity } \\
\text { gas lines }\end{array}$ \\
\hline $\mathrm{U}, \mathrm{Ne}$ & 1538 & 188 & 15 \\
$\mathrm{U}, \mathrm{Ar}$ & 1480 & 179 & 31 \\
\hline
\end{tabular}

We plot the histogram of the number of confirmed $U$ lines as a function of wavelength in Fig. 6. We overplot the high 


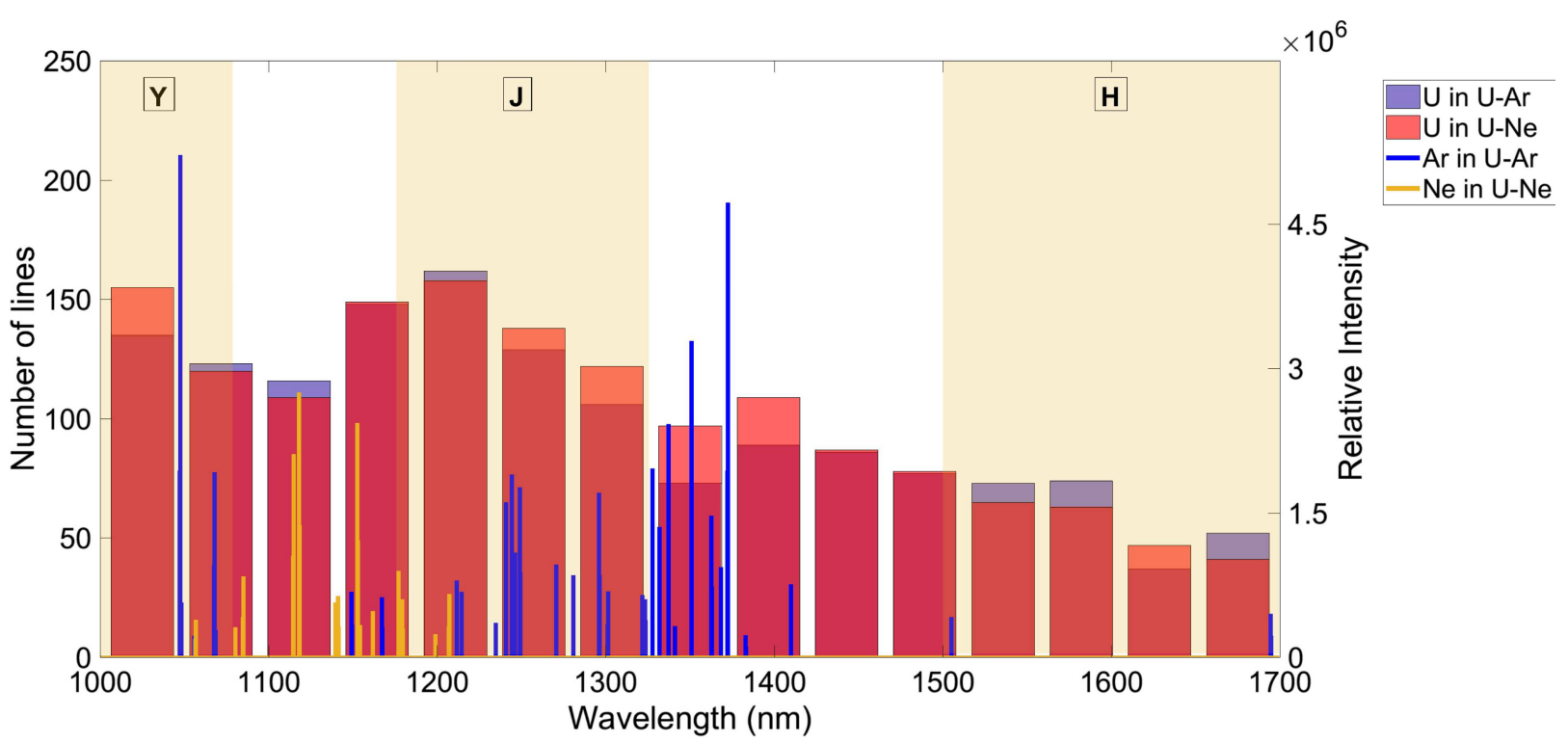

Fig. 6. Same as in Fig. 4 but for NIR wavelengths where only U HCLs (U-Ne and U-Ar) have been studied. In light yellow we indicate the astronomical $Y, J$ and $H$ bands.

Table 3. Molecular bands emitted by the Th-Ar HCL identified in the spectrum recorded using the VIS setup.

\begin{tabular}{cc}
\hline \hline$\lambda_{\mathrm{s}}(\mathrm{nm})$ & $\lambda_{\mathrm{e}}(\mathrm{nm})$ \\
\hline 612.31 & 612.48 \\
689.60 & 691.56 \\
693.05 & 696.53 \\
727.64 & 728.71 \\
787.03 & 791.64 \\
897.42 & 905.06 \\
942.31 & 946.52 \\
\hline
\end{tabular}

Notes. We define $\lambda_{\mathrm{s}}$ and $\lambda_{\mathrm{e}}$ as the start and end wavelength (measured in vacuum) of the molecular band.

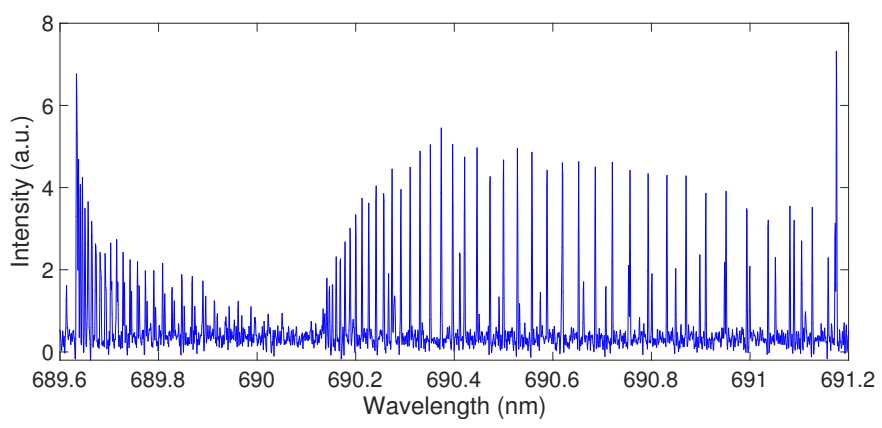

Fig. 7. Example of molecular band observed in the Th-Ar HCL spectrum. See Table 3 for a complete list.

intensity lines emitted by the buffer gas of the lamps as vertical lines.

As seen in Fig. 6, the metal line distributions of both lamps are fairly comparable. We can see a small dip of the line density around $1100 \mathrm{~nm}$ as a result of the lack of sensitivity in the instrument used to prepare the R2011 line list that is used to identify $U$ lines in this section (as explained in Sect. 1). It is interesting to note that the number of high intensity gas lines in the astronomical $Y, J$, and $H$ bands (indicated in light yellow in Fig. 6) is higher in the U-Ar HCL than in the U-Ne.

Since the number of metal lines and their distribution are comparable, but the number of high intensity gas lines is lower in the U-Ne lamps, we conclude that for the spectral range between 1000 and $1700 \mathrm{~nm}$, U-Ne HCLs are more suitable for wavelength calibration of high resolution astronomical spectrographs than U-Ar HCLs.

\section{Molecular contamination}

In the analysis described in the Sect. 4 we explained that we did not detect significant molecular contamination from other species. Nevertheless, we did find seven molecular bands distributed from 612 to $947 \mathrm{~nm}$ of the Th-Ar lamp spectrum (see Table 3). These molecular bands are a source of noise for wavelength calibration. In the case of the Th-Ar HCL, they can contaminate up to $7.64 \mathrm{~nm}$ (e.g. molecular band identified from 897.42 to $905.06 \mathrm{~nm}$ ) of the spectrum. In Fig. 7, the molecular band identified from 689.61 to $691.56 \mathrm{~nm}$ is shown as an example. We observed these features in the new Th-Ar HCLs; we did not find any similar features in our U lamps and Th-Ne HCL.

\section{Comparison of $12 \mathrm{U}-\mathrm{Ne} \mathrm{HCLs}$}

HCLs can show different properties depending on the manufacturer and the manufacturing procedure. The number of emission lines, line intensity ratio between metal and gas lines, and buffer gas contamination have an effect on the performance of HCLs as wavelength calibrators. We analysed the consistency of these characteristics for 12 U-Ne HCLs with the NIR setup. All lamps are from the same batch and have consecutive serial numbers. For each lamp we co-added 120 individual scans at a resolution of $0.035 \mathrm{~cm}^{-1}$. The lamps were operated at $6 \mathrm{~mA}$ resulting in lower $\mathrm{S} / \mathrm{N}$ spectra than in the previous sections. We finely aligned each lamp before its measurement with a power-meter (see Sect. 2).

First, we searched for $\mathrm{U}$ and $\mathrm{Ne}$ lines, and for gas contaminants in the lamps as explained in Sect. 3.2. We did not identify any significant gas contamination from other species in any of 
Table 4. Number of confirmed isolated $\mathrm{U}$ and $\mathrm{Ne}$ lines with $\mathrm{S} / \mathrm{N}$ higher than ten found in the $12 \mathrm{U}-\mathrm{Ne}$ spectra for NIR wavelengths.

\begin{tabular}{cccccc}
\hline \hline HCL & & & & & \\
HKH & $\mathrm{UI}_{\mathrm{I}}$ & $\mathrm{U}_{\mathrm{II}}$ & $\mathrm{NeI}$ & $\mathrm{Ne}$ II & $I^{U}$ \\
\hline 0120 & 273 & 11 & 158 & 40 & 0.74 \\
0121 & 377 & 12 & 151 & 39 & 1.08 \\
0122 & 294 & 9 & 153 & 43 & 0.80 \\
0123 & 334 & 12 & 158 & 44 & 0.95 \\
0124 & 351 & 13 & 145 & 39 & 1.04 \\
0125 & 307 & 9 & 162 & 40 & 0.82 \\
0126 & 295 & 13 & 155 & 42 & 0.80 \\
0127 & 503 & 11 & 153 & 40 & 1.39 \\
0128 & 366 & 10 & 144 & 45 & 1.12 \\
0129 & 398 & 12 & 154 & 39 & 1.09 \\
0130 & 315 & 13 & 157 & 42 & 0.83 \\
0131 & 478 & 11 & 143 & 41 & 1.35 \\
\hline
\end{tabular}

The last column contains the average of the $U$ line intensity normalized to the average of its line intensity in the $12 \operatorname{spectra}\left(I^{U}\right)$.

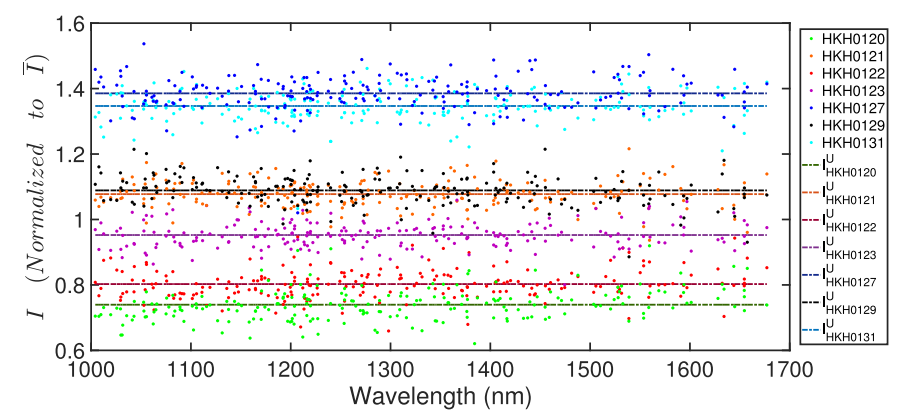

Fig. 8. Coloured dots represent the normalized line intensity of 222 UI lines for each HCL in the spectral range from 1000 to $1700 \mathrm{~nm}$. Dashed coloured lines indicate $I_{\mathrm{HKHi}}^{\mathrm{U}}$ of each lamp (i.e. average of the $222 \mathrm{UI}$ normalized line intensity). For readability reasons we plot only seven of the 12 HCLs.

our spectra. Second, we selected the lines with intensities above a defined threshold (ten times the noise level). We summarize the results in Table 4. We find that the number of Ui lines differs considerably for different lamps (e.g. in the lamp 0127 we find a factor of 1.8 more lines than in the lamp 0120). The number of UII, NeI, and NeII lines is essentially constant.

We looked for the confirmed lines that can be found in all of the 12 spectra to systematically analyse the difference between the 12 lamps. In total, we detected 222 Ur and 104 Ner lines in the spectra of all lamps. We normalized the 12 spectra to the sum of the Ne lines intensities averaged in each spectrum. Then we divided the line intensity of each $U$ line by the average line intensity of this line in the 12 spectra. Last, we calculated the average of the normalized line intensity of the $222 \mathrm{Ur}$ lines (named $I^{U}$ in Table 4) for each lamp.

In Fig. 8 we plot the normalized line intensity of the common Ur lines in the NIR spectral range for seven HCLs (for readability reasons). The dashed lines represent $I^{U}$ for each HCL. From Fig. 8 and Table 4 it can clearly be seen that $I^{U}$ varies significantly between the lamps. Lamps with the lowest U line intensities are HKH0120 and HKH0122. Lamps with the highest U line intensities are HKH0127 and HKH131. The maximum U line intensity difference is $88 \%$ (with respect to the minimum value), measured between lamps HKH0127 and HKH0120.

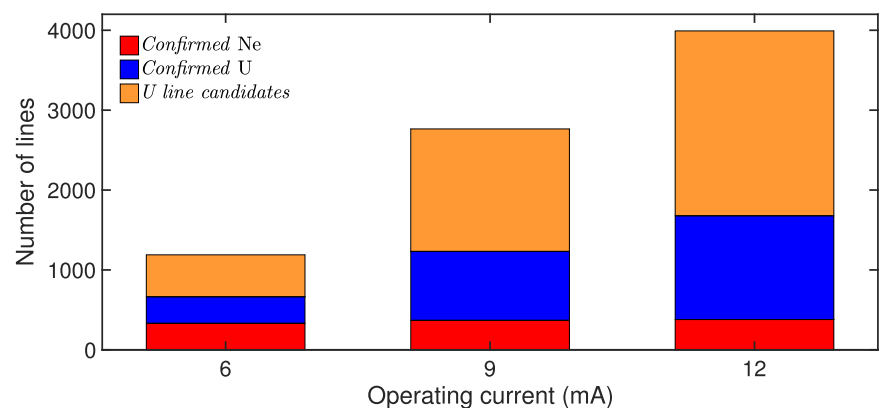

Fig. 9. Number of lines in the spectra recorded when the U-Ne HCL was operated at 6,9 , and $12 \mathrm{~mA}$ using the VIS setup (from 500 to $1000 \mathrm{~nm}$ ). The subset of confirmed U lines (with a corresponding line in the literature) is shown in blue. The subset of confirmed Ne lines is shown in red. We show the number of U line candidates in orange (line candidates with line width comparable to the FWHM of the confirmed $\mathrm{U}$ lines and with no match in the literature).

A possible explanation for the observed behaviour of the HCLs could be a different geometry of the lamps or in the light-fibre coupling efficiency, as seen in Huke et al. (2016). This could affect the gas-metal intensity ratio. Another explanation could be that the metal line intensities are very sensitive to the internal pressure of the buffer gas (Crosswhite et al. 1955). Therefore, even small changes in pressure from lamp to lamp would have an effect on the metal line intensity. We conclude that HCLs can show noticeable differences in number and intensity of metal lines even if they are manufactured in a single batch.

\section{U line list}

We concluded in Sect. 4 that $U$ cathode lamps are more suitable for wavelength calibrators than Th lamps from 500 to $1700 \mathrm{~nm}$. To use HCLs as wavelength calibrators for high precision RV spectrographs, the wavelength of the spectral lines must be known with high accuracy and precision. We present a new line list for $\mathrm{U}$ (ranging from 500 to $1700 \mathrm{~nm}$ ) that complements that of R2011.

\subsection{Line-list in the VIS}

The VIS setup is used to record spectra from 500 to $1000 \mathrm{~nm}$ of one U-Ne HCL operated at 6, 9, and $12 \mathrm{~mA}$. The FTS is set to a resolution of $0.03 \mathrm{~cm}^{-1}$ to record 1000 single scans that are co-added. We identified the confirmed isolated $\mathrm{U}$ and $\mathrm{Ne}$ lines as explained in Sect. 3. The detailed number of lines found in each step in the different spectra is presented in Table A.3 in Appendix A.

In Fig. 9, we plot the number of confirmed $U$ lines (i.e. $U_{I}$ and $\mathrm{U}_{\mathrm{II}}$ ), confirmed $\mathrm{Ne}$ lines (i.e. $\mathrm{Ne}_{\mathrm{I}}$ and $\mathrm{Ne}_{\mathrm{II}}$ ), and $\mathrm{U}$ line candidates, meaning that line candidates with FWHM comparable to the confirmed $U$ lines but no corresponding line in the literature.

In Fig. 9, we see a large increase in the number of confirmed $\mathrm{U}$ lines for higher operational currents while the number of confirmed Ne lines remains almost constant. In order to identify a larger number of $U$ lines, we looked at which of the detected lines have widths comparable to the width of those $U$ lines that are already confirmed. We identify 2314, 1533, and 525 lines in the spectra recorded at 12,9 , and $6 \mathrm{~mA}$ respectively, and flag them as "U line candidates". 


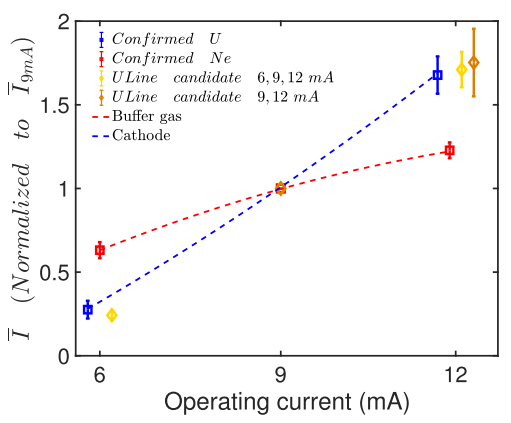

Fig. 10. Weighted average of the line intensity normalized to the line intensity of the line in the $9 \mathrm{~mA}$ spectrum of the four data subsets: 258 confirmed U lines (blue squares) and 194 confirmed Ne lines (red squares), $525 \mathrm{U}$ lines candidates with a match in the 6,9 , and $12 \mathrm{~mA}$ spectra (light brown diamonds), and $1533 \mathrm{U}$ line candidates identified only in the 9 and $12 \mathrm{~mA}$ spectra (brown diamonds). The error bars indicate the weighted standard deviation. The red and blue dashed lines indicate the approximate trend that follows the normalized averaged intensity of the buffer gas and the cathode lines, respectively.

We carried out a second test to check whether our U line candidates are in fact emitted from the $U$ cathode. If so, we expect the intensities of these lines to show a similar behaviour with increasing operational current as the confirmed U lines. We created four data subsets: the confirmed $U$ lines with a line match in the 6,9 , and $12 \mathrm{~mA}$ spectra (258 lines); the confirmed Ne lines with a line match in the 6,9 , and $12 \mathrm{~mA}$ spectra (194 lines); the $\mathrm{U}$ line candidates found in the 6,9, and $12 \mathrm{~mA}$ spectra (525 lines); and the $U$ lines candidates found in 9 and $12 \mathrm{~mA}$ spectra only (1533 lines). Next, we normalized the line intensity of every line to its intensity at $9 \mathrm{~mA}$. Lastly, we calculated the weighted average and the weighted standard deviation of the normalized line intensities for each data subset.

In Fig. 10, we plotted the weighted average and the weighted standard deviation of the four data subsets as a function of the operational current. First, we look at the line intensity of the confirmed $U$ and $\mathrm{Ne}$ lines. We see that the line intensities of these subsets show a different growth rate for different operational currents (see Sect. 1). The line intensity of the U line candidates subsets is consistent with the behaviour of the confirmed $\mathrm{U}$ lines. Because both the FWHM of the $\mathrm{U}$ line candidates and their intensity-rise as a function of operational current are consistent with the confirmed $U$ lines, we conclude that the emitting element of these 1533 lines is indeed $U$.

We provide a line list with the line position measured in vacuum, the line intensity, and the emitting element of all the confirmed and the $\mathrm{U}$ line candidates. Its format is explained in Table 5. The line list is available at the CDS. In addition to the lines discussed above, we find 781 lines that have a FWHM that is consistent with the FWHM of the confirmed U lines. We included these 781 lines in the line list with the flag "12". We also find six lines with FWHM consistent with the width of the confirmed U lines without a corresponding Ritz wavelength. The R2011 line list also contains these lines (where they are flagged as "?"). We checked their line intensity-rise with different operational currents. They show a consistent behaviour with the confirmed $U$ lines. We included these six lines in our line list and flagged them as " $R 2011$ ?".

We analysed the spectral distribution of the U lines. A uniform distribution of lines is desired when choosing a calibrator lamp for astronomical spectrographs. We plot in Fig. 11 the histogram for the number of lines as a function of wavelength of
Table 5. Description of the line list.

\begin{tabular}{lccl}
\hline \hline Column & Symbol & Unit & Explanation \\
\hline 1 & $\tilde{v}_{\mathrm{c}}$ & $\mathrm{cm}^{-1}$ & Line centre in wavenumber \\
2 & $\sigma_{\tilde{v}_{\mathrm{c}}}$ & $\mathrm{cm}^{-1}$ & Uncertainty of the line centre \\
3 & $\lambda$ & $\mathrm{nm}$ & Line centre in wavelength \\
4 & $\sigma_{\lambda}$ & $\mathrm{nm}$ & Uncertainty of the line centre \\
5 & $\mathrm{I}$ & $\mathrm{au}$ & Relative line intensity \\
6 & Species & & Emitting element \\
7 & Notes & & "R2011": contained in R2011 \\
& & & line list. \\
& & & "ULC": U line candidate. \\
& & & at 12 $\mathrm{mA}$ line candidate only \\
& & & "R2011?": U line candidate \\
& & & (“?” in R2011 line list). \\
\hline
\end{tabular}

Notes. The line list is available at the CDS.

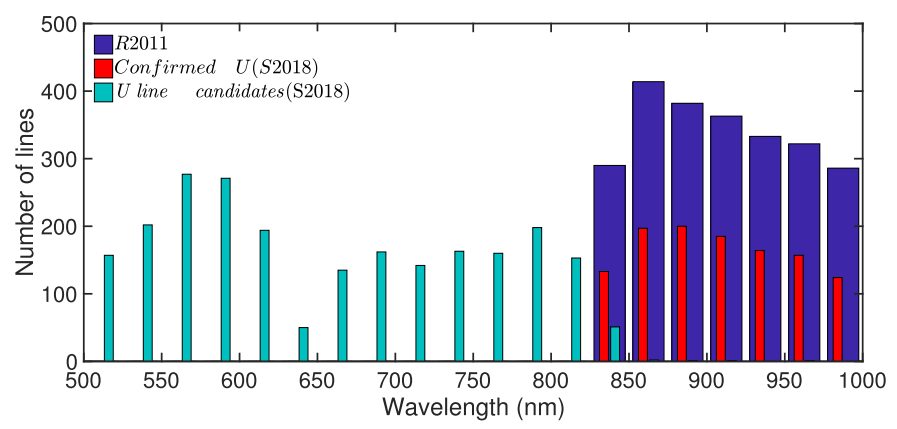

Fig. 11. Uranium line density in the spectral range from 500 to 1000 $\mathrm{nm}$. The blue portion denotes the line list from the literature (R2011). We overplot the confirmed U lines of our work (S2018) in light red. The $\mathrm{U}$ line candidates are denoted in green. The bin size in this plot is constant at $25 \mathrm{~nm}$.

the R2011 line list (blue) and overplot the confirmed U lines in our work (Sarmiento et al. 2018, from now on S2018) contained in R2011 (red) and the U line candidates (green). The shortest wavelength in the R2011 line list is $833 \mathrm{~nm}$, which is the reason why we identify $U$ line candidates bluewards of this wavelength.

When we compare the distribution of lines in the spectral region where our FTS setup overlaps with that of R2011 (blue and red histograms in Fig. 11), we observe that about $50 \%$ of the lines listed in R2011 are identified in our data. We attribute this difference in the number of lines to the different operational current used in this work and R2011. While in R2011 the spectra were taken with operational currents between 26 and $300 \mathrm{~mA}$, we used spectra taken at 6,9 , and $12 \mathrm{~mA}$ and therefore our line list is limited to the brighter spectral features.

The number of identified lines in our spectra (confirmed + U line candidates) decreases towards the blue and red end of the VIS spectral range. We attribute this decrease in number of lines to the spectral response sensitivity of our setup and instrument. In addition, we also observe a low number of lines in the region from 600 to $700 \mathrm{~nm}$. This spectral band is strongly contaminated by the internal FTS He-Ne laser (see Sect. 2), which is why the weaker spectral lines are likely missed.

The spectrum of the U lamp does not show any extended spectral region that is completely devoid of U lines. Overall, the number of spectral lines per $25 \mathrm{~nm}$ bin is relatively constant and varies only up to a factor of two in our line list (except 


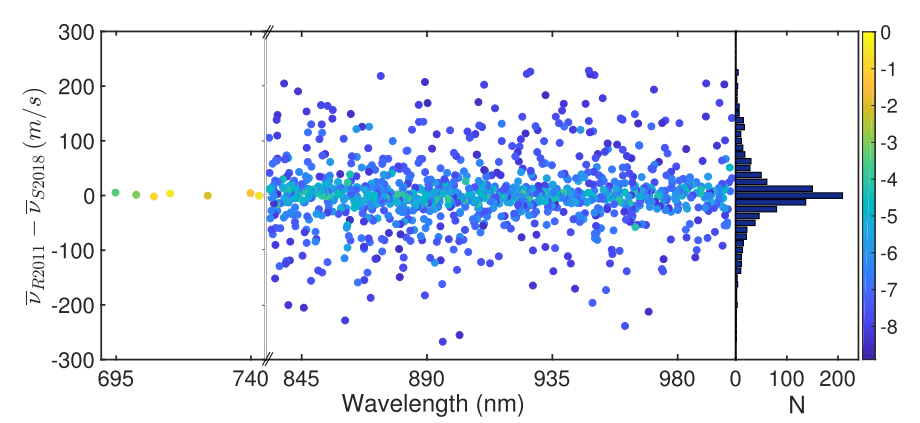

Fig. 12. Left panel: line position difference in velocity units of the isolated confirmed $U$ lines (i.e. with a corresponding match in the literature). The colour scale located at the right edge of the plot denotes the normalized line intensity in a logarithmic scale. From 690 to $744 \mathrm{~nm}$, we can see the residuals of the eight standard lines used to calculate the $\bar{k}_{\mathrm{VIS}}$. The $x$-axis is shortened for readability reasons at $744 \mathrm{~nm}$. Right panel: distribution of the residuals. The distribution is centred on $-0.12 \pm 8.19 \mathrm{~m} \mathrm{~s}^{-1}$, where the uncertainty is calculated as the weighted standard deviation plus the statistical error introduced by the standard lines used for calibration.

for the bin most contaminated by the $\mathrm{He}-\mathrm{Ne}$ laser centred on $637.5 \mathrm{~nm}$ ). The overall number of calibration lines available and their relatively uniform spectral distribution make U lamps good candidates to calibrate astronomical spectrographs operating in optical wavelength regimes.

We investigated possible systematics in the wavelength positions of our line list using the R2011 line list in the overlapping spectral region from 833 to $1000 \mathrm{~nm}$. The left panel of Fig. 12 shows the difference in line position between R2011 and our work; the histogram in the right panel shows the distribution of the residuals as a function of velocity units.

In Fig. 12 we do not see systematics in our work when comparing with the R2011 line list. The mean difference between the two line lists is $-0.12 \pm 8.19 \mathrm{~m} \mathrm{~s}^{-1}$ (weighted mean and standard deviation). We conclude that the line positions in our line list are consistent with those in R2011 within the error bars.

Our VIS line list provides a total of $3480 \mathrm{U}$ lines, of which 2320 lines are $\mathrm{U}$ line candidates. There are 2314 lines that are not contained in R2011 because the line list does not show information in wavelengths bluewards of $833 \mathrm{~nm}$ (plus six U line candidates that are flagged as "R2011?"). We conclude that the even distribution and high abundance of lines render $U$ lamps excellent calibrators for both NIR and VIS range spectrographs.

\subsection{Line-list in the NIR}

We recorded and co-added 450 single scans of the U-Ne lamp at a resolution of $0.01 \mathrm{~cm}^{-1}$, using the NIR setup from 1000 to $1700 \mathrm{~nm}\left(10000\right.$ to $\left.5882.4 \mathrm{~cm}^{-1}\right)$. The U-Ne HCL was operated at three different operational currents 8,10 , and $12 \mathrm{~mA}$ (see Table A.1 for further details). We detected and identified the lines as explained in Sect. 3. In Fig. 13, we plot the number of confirmed lines and U line candidates in the NIR spectra.

As in the VIS setup, we can see in Fig. 13 that the number of confirmed U lines increases substantially at higher operational currents, while the number of confirmed $\mathrm{Ne}$ lines remains constant (see Sect. 1). We see a large number of $U$ line candidates in the three spectra. Since our NIR setup overlaps with the spectral range of R2011, we compared the line position and intensity of our $U$ line candidates and the confirmed U lines (i.e. with a match in R2011 line list). In Fig. 14 we plot the relative intensity

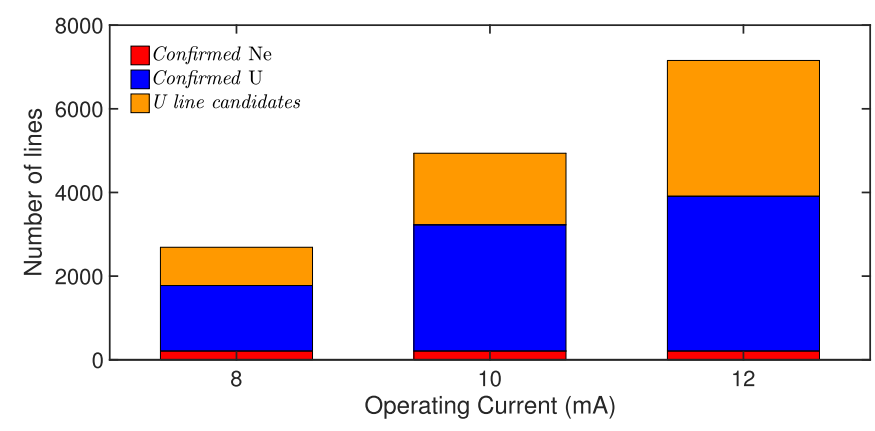

Fig. 13. Same as in Fig. 9, but for NIR wavelengths (from 1000 to $1700 \mathrm{~nm}$ ), when the U-Ne HCL was operated at 8, 10, and $12 \mathrm{~mA}$.

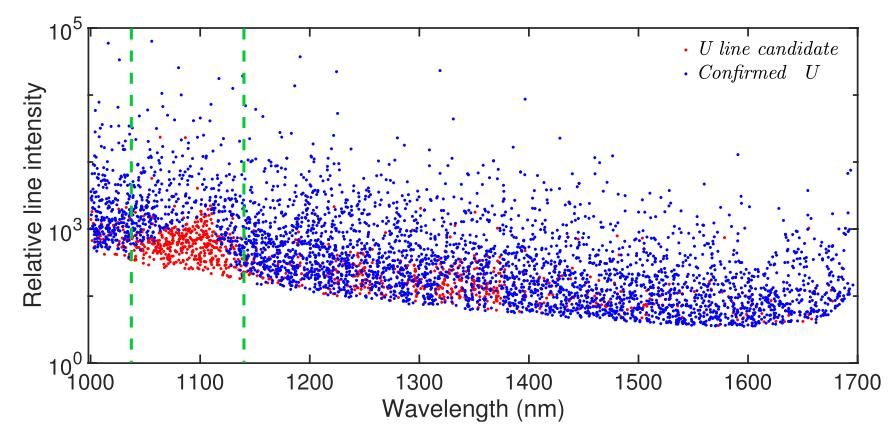

Fig. 14. Relative line intensity of the $U$ line candidates (red dots) and the confirmed $U$ lines (blue dots) as a function of wavelength. The green dashed lines indicate the spectral band from 1031 to $1139 \mathrm{~nm}$, where the R2011 line list shows a lack of emision lines caused by the spectral response of its spectrograph.

of the $\mathrm{U}$ line candidates (red dots) and overplot the confirmed $\mathrm{U}$ lines (blue dots) found in both the $10 \mathrm{~mA}$ and the $12 \mathrm{~mA}$ spectra.

Two spectral regions in Fig. 14 are of special interest: (i) in the range from 1031 to $1139 \mathrm{~nm}$ (indicated by the green dashed lines), the R2011 line list is lacking a number of lines that we found in our spectra, and (ii) there are several narrow bands between 1200 and $1400 \mathrm{~nm}$ where our spectra show U line candidates that are close to strong Ar lines (see Fig. 6).

We carried out a second test to determine the emitting element of our $U$ line candidates following the methodology indicated in Sect. 7.1. We first created four data subsets; 1418 confirmed U lines found in all spectra (blue squares); 132 confirmed $\mathrm{Ne}$ lines contained in all spectra (red squares); $137 \mathrm{U}$ line candidates found in all spectra (light brown diamonds); and $908 \mathrm{U}$ line candidates identified in the spectra of 10 and $12 \mathrm{~mA}$ (brown diamonds). We normalized the intensity of every line to its intensity at $10 \mathrm{~mA}$, and we calculated the weighted average of the normalized line intensity of each data subset. We plot the results in Fig. 15.

Figure 15 shows that the line intensities of both the confirmed $\mathrm{U}$ and Ne lines (blue and red squares, respectively) show different behaviour for different operational currents. We find that the growth in line intensity of both sets of $U$ line candidates is consistent with the behaviour of the confirmed $U$ lines. Because the FWHM of the $\mathrm{U}$ line candidates is also consistent with the width of the confirmed U lines, we conclude that these 908 lines are U lines. We also find 151 lines with FWHM consistent with the width of the confirmed U lines (flagged as "?" in R2011). For these lines, no corresponding Ritz wavelength is available in R2011. We confirm that these are $\mathrm{U}$ lines because they show the same behaviour with operational currents as the 


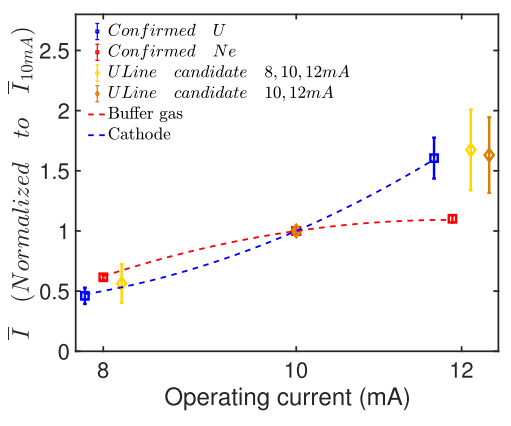

Fig. 15. Same as in Fig. 10, but when the U-Ne lamp is operated at 8,10 , and $12 \mathrm{~mA}$ and the line intensity is normalized to its intensity at $10 \mathrm{~mA}$.

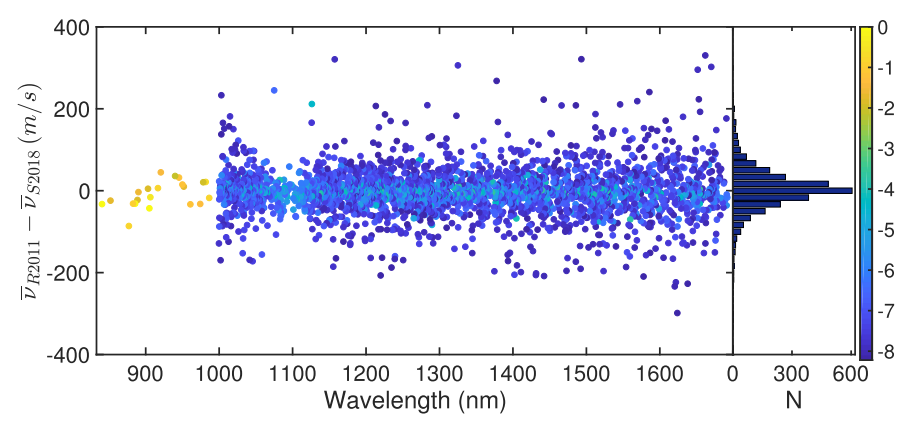

Fig. 16. Same as Fig. 12 but from 833 to $1700 \mathrm{~nm}$. Left panel: the wavelength of the 21 lines used to calculate $\bar{k}_{\mathrm{NIR}}$ factor are from 833 to $1000 \mathrm{~nm}$. Right panel: the distribution is centred on $-3.5 \pm 11.0 \mathrm{~m} \mathrm{~s}^{-1}$.

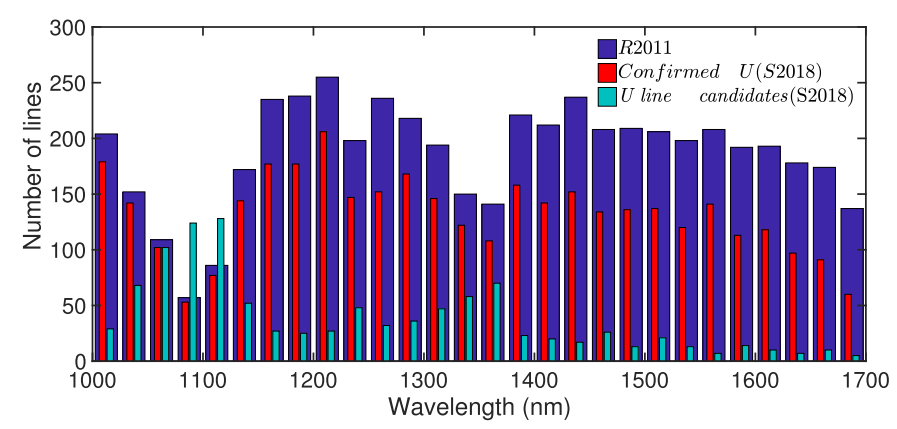

Fig. 17. Same as Fig. 11, but for NIR wavelengths from 1000 to $1700 \mathrm{~nm}$.

confirmed U lines with Ritz wavelengths. We included these 151 lines in our line list with the flag "R2011?".

We investigated possible systematics introduced by our NIR setup using the R2011 line list. The left panel of Fig. 16 shows the difference in line position between our S2018 and the R2011 line lists. There are no obvious systematic differences between the two lists; higher intensity lines show less scatter than the lower intensity lines. In the right panel of Fig. 16, the histogram shows the distribution of the differences. The mean difference between the two line lists is $-3.5 \pm 11.0 \mathrm{~m} \mathrm{~s}^{-1}$ (weighted mean and standard deviation), that is, the two distributions are consistent within the uncertainties.

The spectral distribution of $U$ lines in our line list obtained with the NIR setup is shown in Fig. 17 with U lines of the R2011 line list (blue), of our confirmed $U$ lines (red), and of our $U$ line candidates (green). The U lines are distributed across the entire NIR wavelength range rather homogeneously. Two spectral regions stand out in Fig. 17 (see also Fig. 14): (i) from 1031 to
$1139 \mathrm{~nm}$, the R2011 line list contains relatively few lines; and (ii) the spectrum is contaminated by high intensity Ar lines (see Fig. 6). In the spectral region from 1031 to $1139 \mathrm{~nm}$, our line list almost doubles the number of known U lines: in R2011, 424 $\mathrm{U}$ lines are listed between 1031 to $1139 \mathrm{~nm}$. While we identify 847 lines in this band, 444 of them $U$ line candidates. We see a decrease in the number of lines in the spectral range around $1230 \mathrm{~nm}$ and around $1340 \mathrm{~nm}$ in the R2011 line list, where we identify $86 \mathrm{U}$ and $218 \mathrm{U}$ line candidates, respectively.

\section{Summary}

This work presents the analysis of four HCLs with different combinations of cathode material and buffer gas. We recorded spectra of U-Ne, U-Ar, Th-Ne, and Th-Ar lamps, compared their performance, and created a new $U$ line list for the calibration of high resolution VIS and NIR spectrographs.

We found that in the VIS, the U lamps exhibited a significantly larger number of spectral lines than the Th lamps. For the lamps filled with Ar, the difference was about 30\%. The difference was more severe in lamps filled with Ne. Here, the U cathode provided about a factor of two more lines than the Th cathode. The total number of lines was comparable for the two U cathode lamps; the one filled with Ar showed about 5\% more lines in the VIS wavelength range. This difference was very large for the Th lamps where the lamp filled with Ar had about 50\% more lines than the one filled with $\mathrm{Ne}$.

Our analysis of the spectral line distribution demonstrated that the combination of a U-Ne lamp and a U-Ar lamp can map out the wavelength range of VIS spectrographs very efficiently. This strategy can provide a high number of well defined cathode lines and mitigate the effect of the pixel saturation because the strong gas lines from $\mathrm{Ar}$ and $\mathrm{Ne}$ contaminate different wavelength ranges.

At NIR wavelengths, we only investigated $U$ cathode lamps because Th lamps are known to show fewer lines here. The U-Ne and U-Ar lamps showed comparable performances that are very useful for wavelength calibration. The Ne lamp spectrum showed fewer strong lines and might therefore be preferred over the lamps filled with Ar.

As a product of our analysis, we presented a line list for $U$ in the wavelength range $500-1700 \mathrm{~nm}$. The catalogue contains 8239 unblended lines that we attributed to U. The line list allows the calibration of high resolution spectrographs with $U$ cathode lamps. It can be expected to improve the wavelength calibration accuracy because of the larger number of lines, and it provides an alternative to Th lamps also for visual light spectrographs.

Acknowledgements. LFS acknowledges support from the European Research Council under FP7 GA 279347 and AY2011-30147-C03-03.

\section{References}

Conway, J. G., Worden, E. F., Brault, J. W., Hubbard, R. P., \& Wagner, J. J. 1984, Atomic Data Nuclear Data Tables, 31, 299

Crosswhite, H. M., Dieke, G. H., \& Legagneur, C. S. 1955, J. Opt. Soc. Am., 45, 270

Degraffenreid, W., \& Sansonetti, C. J. 2002, J. Opt. Soc. Am. B Opt. Phys., 19, 1711

Dieke, G. H., \& Crosswhite, H. M. 1952, J. Opt. Soc. Am., 42, 433

Engleman, R. J., Hinkle, K. H., \& Wallace, L. 2003, J. Quant. Spectr. Rad. Transf., 78, 1

Fischer, D. A., Anglada-Escude, G., Arriagada, P., et al. 2016, PASP, 128

Giacchetti, A., Blaise, J., Corliss, C., \& Zalubas, R. 1974, J. Res. Nat. Bur. Stand., 2, 247 
Griffiths, P. R., \& de Haseth, J. A. 2007, Fourier Transform Infrared Spectrometry (Hoboken: Wiley)

Huke, P., Tal-Or, L., Sarmiento, L. F., \& Reiners, A. 2016, in Proc. SPIE, Advances in Optical and Mechanical Technologies for Telescopes and Instrumentation II, 9912, 99124K

Jones, W. G., \& Walsh, A. 1960, Spectrochim. Acta, 16, 249

Kerber, F., Nave, G., Sansonetti, C. J., Bristow, P., \& Rosa, M. R. 2007, in The Future of Photometric, Spectrophotometric and Polarimetric Standardization ASP, ed. Sterken (Berlin, Heidelberg: Springer), 364, 461

Kerber, F., Nave, G., \& Sansonetti, C. J. 2008, A\&AS, 178, 374

Kramida, A., Yu. R., Reader, J., \& NIST ASD Team 2015, NIST Atomic Spectra Database (ver. 5.3), http://physics.nist.gov/asd [2016, April 1]. (National Institute of Standards and Technology, Gaithersburg, MD)

Lieberman, M., \& Lichtenberg, A. 1994, Mater. Corrosion, 46, 551

Lovis, C., \& Pepe, F. 2007, A\&A, 468, 1115

Mayor, M., Pepe, F., Queloz, D., et al. 2003, The Messenger, 114, 20

Palmer, B. A., \& Engleman, J. R. 1983, Los Alamos National Laboratory Report, LA-9615
Palmer, B. A., Keller, R. A., \& Engleman, R. 1980, Los Alamos Scientific Laboratory, Report number LA-8251-MS

Paschen, F. 1916, Ann. Phys., 355, 901

Quirrenbach, A., Amado, P. J., Caballero, J. A., et al. 2014, in Proc. SPIE, Ground-based and Airborne Instrumentation for Astronomy V, 9147, 91471F

Redman, S. L., Lawler, J. E., Nave, G., Ramsey, L. W., \& Mahadevan, S. 2011, ApJ, 195, 24

Redman, S. L., Nave, G., \& Sansonetti, C. J. 2014, ApJ, 211, 4

Reiners, A., Bean, J. L., Huber, K. F., et al. 2010, ApJ, 710, 432

Rosman, K. J. R., \& Taylor, P. D. P. 1998, J. Phys. Chem. Ref. Data, 27, 1275

Russell, B. J., Shelton, J. P., \& Walsh, A. 1957, Spectrochim. Acta, 8, 317

Stanley, R. W., \& Dieke, G. H. 1955, J. Opt. Soc. Am., 45, 280

Tolansky, S. 1947, High Resolution Spectroscopy (London: Methuen)

Zalubas, R. 1976, J. Res. Nat. Bur. Stand., NBS A, 221

Zalubas, R., \& Corliss, C. 1974, J. Res. Nat. Bur. Stand., NBS A, 163 
L. F. Sarmiento et al.: Comparing the emission spectra of $U$ and Th hollow cathode lamps and a new $U$ line list

\section{Appendix A: Summary of the different measurements and complementary figures}

Table A.1. Table of the different measurements.

\begin{tabular}{lcccccc}
\hline \hline Experiment & $\begin{array}{c}\text { HCL } \\
\text { elements }\end{array}$ & $\begin{array}{c}\text { Operational } \\
\text { current }(\mathrm{mA})\end{array}$ & $\begin{array}{c}\text { Resolution } \\
\left(\mathrm{cm}^{-1}\right)\end{array}$ & $\begin{array}{c}\text { Scanning } \\
\text { Time }(\mathrm{h})\end{array}$ & $\begin{array}{c}\text { Spectral } \\
\text { \# Scans }\end{array}$ & \begin{tabular}{c} 
range \\
\hline Properties of the U HCLs
\end{tabular} \\
U,Ne & 6 & 0.035 & 1.9 & 120 & NIR \\
\hline Cathode selection & U,Ne & 12 & 0.03 & 2.7 & 200 & Vis \\
& U,Ar & 12 & 0.03 & 2.7 & 200 & Vis \\
& Th,Ne & 12 & 0.03 & 2.7 & 200 & Vis \\
& Th,Ar & 12 & 0.03 & 2.7 & 200 & Vis \\
& U,Ne & 12 & 0.02 & 3.3 & 200 & NIR \\
& U,Ar & 12 & 0.02 & 3.3 & 200 & NIR \\
\hline Line list & U,Ne & 6 & 0.03 & 13.7 & 1000 & Vis \\
& U,Ne & 9 & 0.03 & 13.7 & 1000 & Vis \\
& U,Ne & 12 & 0.03 & 13.7 & 1000 & Vis \\
& U,Ne & 8 & 0.01 & 22.6 & 450 & NIR \\
& U,Ne & 10 & 0.01 & 22.6 & 450 & NIR \\
& U,Ne & 12 & 0.01 & 22.6 & 450 & NIR \\
\hline
\end{tabular}

Table A.2. Summary of the line identification for the experiment: cathode selection.

\begin{tabular}{lccccccc}
\hline \hline Experiment & $\begin{array}{c}\text { HCL } \\
\text { elements }\end{array}$ & $\begin{array}{c}\text { \# Detected } \\
\text { lines }\end{array}$ & $\begin{array}{c}\text { Metal } \\
\text { lines }\end{array}$ & $\begin{array}{c}\text { Not blended } \\
\text { metal lines }\end{array}$ & $\begin{array}{c}\text { Gas } \\
\text { lines }\end{array}$ & $\begin{array}{c}\text { Not blended } \\
\text { gas lines }\end{array}$ & $\begin{array}{c}\text { Spectral } \\
\text { range }\end{array}$ \\
\hline Cathode and & U,Ne & 8690 & 2256 & 1899 & 274 & 235 & Vis \\
buffer gas & U,Ar & 9227 & 2374 & 1998 & 240 & 195 & Vis \\
selection & Th,Ne & 6001 & 1044 & 938 & 270 & 231 & Vis \\
& Th,Ar & 8323 & 1641 & 1436 & 275 & 233 & Vis \\
& U,Ne & 4901 & 2080 & 1538 & 351 & 188 & NIR \\
& U,Ar & 7431 & 1943 & 1480 & 315 & 179 & NIR \\
\hline
\end{tabular}

Table A.3. Line identification for the experiment: line list in the VIS spectral range.

\begin{tabular}{lcccccccccc}
\hline \hline $\begin{array}{l}\text { Operational } \\
\text { current (mA) }\end{array}$ & $\begin{array}{c}\text { \# Detected } \\
\text { lines }\end{array}$ & $\mathrm{U}_{I}$ & $\mathrm{U}_{I}^{I s o}$ & $\mathrm{U}_{I I}$ & $\mathrm{U}_{I I}^{I s o}$ & $\mathrm{Ne}_{I}$ & $\mathrm{Ne}_{I}^{I s o}$ & $\mathrm{Ne}_{I I}$ & $\mathrm{Ne}_{I I}^{I s o}$ & $\mathrm{U}_{\text {Candidates }}$ \\
\hline 12 & 12378 & 1268 & 1132 & 31 & 28 & 248 & 229 & 131 & 124 & 2314 \\
9 & 11602 & 845 & 749 & 18 & 16 & 244 & 225 & 125 & 110 & 1533 \\
6 & 8969 & 329 & 274 & 3 & 2 & 232 & 212 & 100 & 75 & 525 \\
\hline
\end{tabular}

Table A.4. Line identification for the experiment: line list in the NIR spectral range.

\begin{tabular}{ccccccccccc}
\hline \hline $\begin{array}{c}\text { Operational } \\
\text { current (mA) }\end{array}$ & $\begin{array}{c}\text { \# Detected } \\
\text { lines }\end{array}$ & $\mathrm{U}_{I}$ & $\mathrm{U}_{I}^{I s o}$ & $\mathrm{U}_{I I}$ & $\mathrm{U}_{I I}^{I s o}$ & $\mathrm{Ne}_{I}$ & $\mathrm{Ne}_{I}^{I s o}$ & $\mathrm{Ne}_{I I}$ & $\mathrm{Ne}_{I I}^{I s o}$ & $\mathrm{U}_{\text {Candidates }}$ \\
\hline 12 & 8723 & 3982 & 3550 & 169 & 150 & 192 & 160 & 62 & 49 & 3246 \\
10 & 6211 & 3269 & 2942 & 81 & 74 & 191 & 160 & 62 & 49 & 1713 \\
8 & 3306 & 1731 & 1549 & 19 & 17 & 189 & 157 & 52 & 39 & 916 \\
\hline
\end{tabular}



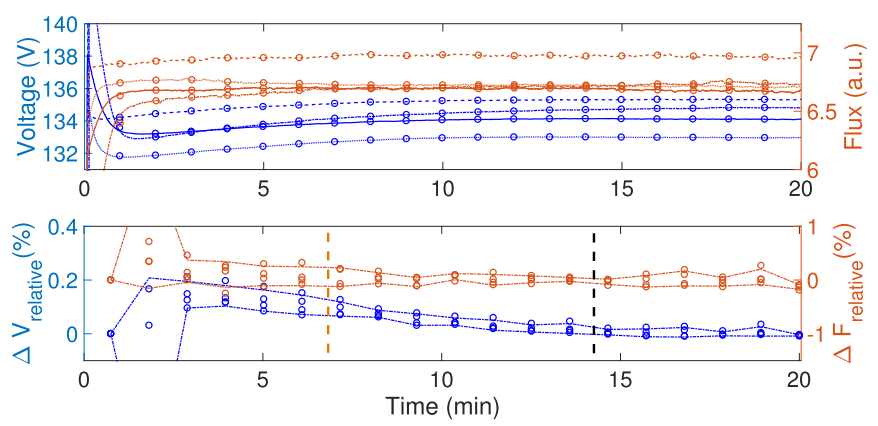

Fig. A.1. Voltage and flux measurement during warm-up time of the four different HCLs. Upper panel: the blue lines indicate voltage measurements ( $y$-axis in the left) and the brown lines represent the flux ( $y$-axis in the right). Blue and brown circles indicate the one minute average values. Bottom panel: the left axis indicates the relative voltage variation, and the right axis the relative flux variation. Blue and brown circles represent voltage and flux respectively. Blue and brown lines indicate $\pm \sigma$ of the calculated relative variation for voltage and flux. The brown dashed vertical line (at about seven minutes) indicates when the flux reaches a stability of $0.5 \%$ for all the lamps. The black vertical dashed line indicates when the voltage is stable with relative changes lower than $0.05 \%$. We consider that the lamps offer their most stable output after $14-15 \mathrm{~min}$.

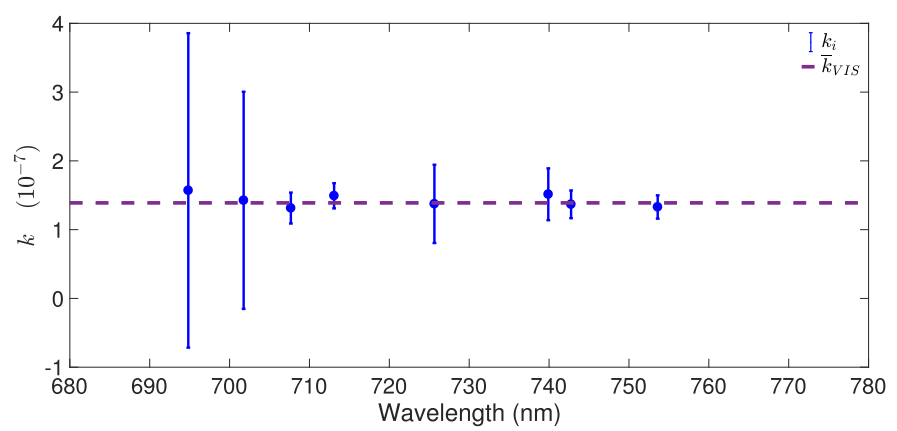

Fig. A.2. FTS $k$ factor used to obtain an absolute wavelength calibration of the spectra recorded from 500 to $1000 \mathrm{~nm}$. We use eight U standard lines from Degraffenreid et al. (2002) that have a match in our spectrum. The blue dots indicate the $k_{\mathrm{i}}$ of each line. The error bars indicate the uncertainty calculated by error propagation of the line position measurement of our lines and the standard lines. The dashed purple line indicates the weighted average of the $8 k_{\mathrm{i}}$.

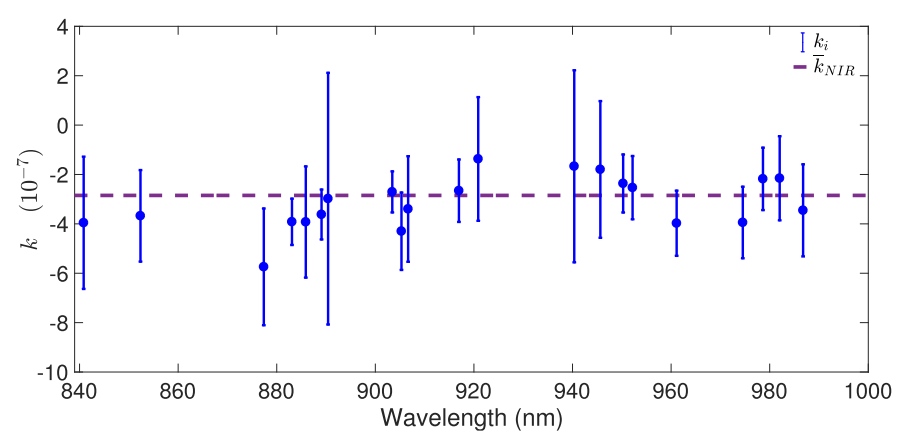

Fig. A.3. As in Fig. A.2, but for the NIR spectrum. We use 21 high intensity isolated lines to calculate each $k_{\mathrm{i}}$ confirmed in the VIS and in the NIR spectrum. 